\title{
Uptake of Photosensitizer 2-Devinyl-2-(1-methoxylethyl) Chlorin $f$ in Human Breast Cancer Cells: A Diffusion Kinetics Study
}

\author{
Ping Chen, ${ }^{1}$ Feng Zhang,, Lei Zhang, ${ }^{1}$ Song-Cheng Mao, ${ }^{1}$ Lie Lin, ${ }^{1}$ Hua Bai, ${ }^{1}$ \\ Guo-Qing Tang, ${ }^{1}$ Jian-Zhong Yao, ${ }^{2}$ Jun-Hong Yang, ${ }^{3}$ and Yun-Feng $\mathrm{Ma}^{3}$ \\ ${ }^{1}$ Institute of Modern Optics, Nankai University, Tianjin 300071, China \\ ${ }^{2}$ School of Pharmacy, Second Military Medical University, Shanghai 200433, China \\ ${ }^{3}$ R\&D Department, Beijing GK Laser Technology Co., Ltd., No. 66 Xixiaokou Road, Beijing 100192, China
}

Correspondence should be addressed to Lei Zhang, lzzhang@nankai.edu.cn and Jian-Zhong Yao, yaojz@sh163.net

Received 13 July 2011; Accepted 10 October 2011

Academic Editor: Peter Robertson

Copyright (c) 2012 Ping Chen et al. This is an open access article distributed under the Creative Commons Attribution License, which permits unrestricted use, distribution, and reproduction in any medium, provided the original work is properly cited.

\begin{abstract}
The kinetics of photosensitizer 2-devinyl-2-(1-methoxylethyl) chlorin $f$ (CPD4) uptake in MCF-7 human breast cancer cells is described by a diffusion kinetics model and experimentally investigated using laser scanning confocal microscopy (LSCM). CPD4 permeated into MCF-7 cells with increasing incubation time, which was followed by its binding to cell organelles. Subcellular distribution study revealed that CPD4 was primarily localized on the mitochondria and membranes, supporting that the mode of transmembrane transport was diffusion. A kinetics model describing CPD4 passing through the plasma membrane of MCF-7 cells was proposed based on Fick's first law of diffusion. The kinetics of cellular uptake of CPD4 was studied by three-dimensional LSCM. By fitting the experimental data using the above model, important cellular uptake and distribution parameters were obtained, which are of clinical significance in photodynamic therapy.
\end{abstract}

\section{Introduction}

Photodynamic therapy (PDT) is a promising therapeutic technique for the treatment of cancerous or other unwanted tissues based on the combined use of light irradiation and photosensitizer (PS) [1-9]. After photoactivation of PS, the interaction between the excited triplet PS and ground-state triplet oxygen $\left({ }^{3} \mathrm{O}_{2}\right)$ generates singlet oxygen $\left({ }^{1} \mathrm{O}_{2}\right)$, which is the main cytotoxic species that can lead to photodynamic damage of tumor cells and tissues [10-16]. In comparison with surgical resection followed by radio- and/or chemotherapy, PDT offers the advantage of being able to siteselectively destroy diseased tissues with minimal damage to the surrounding healthy tissues [17-21]. These outstanding features pave the way for clinical application of PDT in many developed or developing countries.

Photodynamic damage can be only induced in the close vicinity of ${ }^{1} \mathrm{O}_{2}$, because ${ }^{1} \mathrm{O}_{2}$ in cells has a short lifetime $(<40 \mathrm{~ns})$ and a limited diffusion in space $(<20 \mathrm{~nm})$ [22]. This indicates that the efficacy of PDT is highly dependent on the amount and distribution of intracellular PS [23]. To evaluate the photodynamic activity of a certain PS, two properties need to be addressed. The first is the intracellular accumulation level of ${ }^{1} \mathrm{O}_{2}$, which relies on both the concentration of PS and the quantum yield of ${ }^{1} \mathrm{O}_{2}$ production. The second is the subcellular distribution of PS, which is a crucial parameter influencing the extent of cell damage, as well as cell response and the mechanism of the resultant cell death. From a kinetic point of view, the binding between PS and organelles could also lead to loading more PS into the cells by means of maintaining a concentration gradient beneficial to transport of PS from the outside to the inside of cells and meanwhile suppressing the reverse process.

Previous investigations have shown that the partition of PS among subcellular structures is most likely dependent on the dynamics of its transport through membranes [24-28]. For example, photofrin II permeated into cells by diffusion and then localized on the mitochondria, while mono- $L$ aspartyl chlorin $e_{6}$ was internalized into cells via endocytosis and then accumulated on the lysosomes [25]. Thus, revealing the subcellular distribution of PS is useful for determining 
the transmembrane transport modes, which provides the basis for the development of physical models toward quantitative understanding of the transport process. The use of physical models to obtain novel information about biological processes has been reported in the literature. For example, to estimate the threshold dose of ${ }^{1} \mathrm{O}_{2}$ for tissue necrosis, Stratonnikov et al. proposed a model to describe the oxygen consumption and photobleaching in whole blood samples incubated with PS [29]. Quantitative analyses of molecular motions in cells were also successfully carried out based on the use of diffusion models $[30,31]$. In this contribution, we aim at establishing a model to quantitatively describe the transmembrane transport process of PS.

Most of the PSs currently used in research and clinics are derived from the tetrapyrrole aromatic ring. First-generation PSs include hematoporphyrin derivative (HpD), while second-generation PSs are composed of metalloporphyrins, porphycenes, pheophorbides, purpurins, chlorins, protoporphyrin, and phthalocyanines [3]. Chlorins belong to the second-generation PDT sensitizers and are characterized by the presence of one reduced peripheral double bond of the tetrapyrrole ring, leading to a strong absorption of red light $\left(\lambda_{\max }=650-680 \mathrm{~nm}\right)$, where tissue transparency is optimal [32]. Prominent features of chlorins also include efficient triplet formation at high energy to allow ${ }^{1} \mathrm{O}_{2}$ production, absence of dark toxicity, and selective retention in the selected target tissue combined with rapid clearance from the body [33]. Chlorophyll $a, b$, and $c$ are the most abundant among the naturally occurring tetrapyrrolic pigments and serve as the starting materials for the preparation of various chlorins [34]. The PS used in this work is 2-devinyl-2-(1methoxylethyl) chlorin $f$ (hereafter abbreviated as CPD4, Figure 1), which is a chlorophyll derivative synthesized from the extract of silkworm excrement [35]. Attractive properties of CPD4 have been disclosed by spectral measurements, ${ }^{1} \mathrm{O}_{2}$ quantum yield determination, and photoinduced damage studies [36-38]. In this paper, the process of CPD4 uptake in MCF-7 human breast cancer cells is described by a diffusion kinetics model and experimentally investigated using laser scanning confocal microscopy (LSCM).

\section{Experimental Section}

2.1. Photosensitizer and Cell Culture. CPD4 was synthesized from the extract of silkworm feces with the purity greater than $98 \% \cdot 10^{-4} \mathrm{M}$ of CPD4 stock solution was prepared by dissolving CPD4 powder in $0.1 \mathrm{M} \mathrm{NaOH}$ solution. MCF7 human breast cancer cells were offered by the Cancer Hospital of Tianjin Medical University, Tianjin, China. MCF7 cells were grown in RPMI 1640 supplemented with $10 \%$ fetal bovine serum (FBS) and 1\% penicillin/streptomycin (Gibco BRL, USA) and were cultured under the atmosphere of $5 \% \mathrm{CO}_{2}$ and $95 \%$ air at $37^{\circ} \mathrm{C}$.

2.2. Subcellular Distribution of CPD4 in MCF-7 Cells. $4 \times$ $10^{4}$ MCF-7 cells were plated into two $35 \mathrm{~mm}$ cell culture dishes (Corning, USA). CPD4 was added to each dish to a final concentration of $4.4 \times 10^{-6} \mathrm{M}$ and incubated with cells for 2 hours. Rhodamine 123 (Sigma, USA) was added

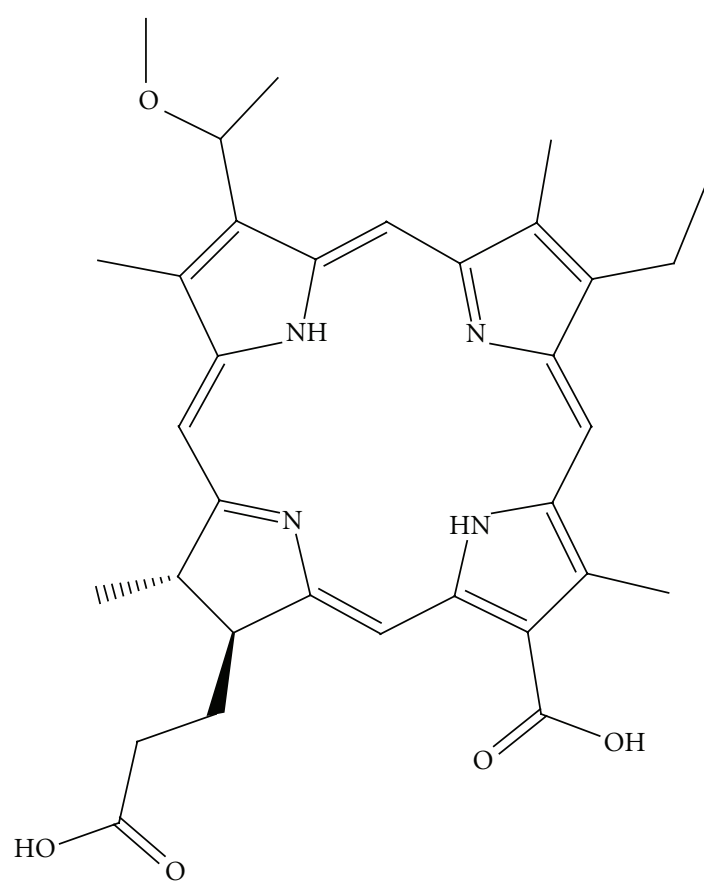

Figure 1: Molecular structure of CPD4.

to a dish to a final concentration of $25 \mathrm{nM}$ and incubated for $15 \mathrm{~min}$; DiI-7 (Sigma, USA) was added to a second dish to a final concentration of $20 \mathrm{nM}$ and incubated for $30 \mathrm{~min}$. Using LSCM (Olympus FV300, Olympus Co., Tokyo, Japan), the samples were excited by $488 \mathrm{~nm} \mathrm{Ar}^{+}$laser (for CPD4 and Rhodamine 123) and $543 \mathrm{~nm} \mathrm{He}-\mathrm{Ne}$ laser (for CPD4 and DiI-7), respectively. Combinations of dichroic mirrors and narrow bandpass filters were used for the detection of fluorescence. The $40 \mathrm{x}$ objective was used to visualize features smaller than $30 \mu \mathrm{m}$. LSCM images were scanned at $1.65 \mathrm{~s} /$ frame and each image contained $512 \times 512$ pixels.

2.3. Kinetics of CPD4 Uptake in MCF-7 Cells. $4 \times 10^{4}$ MCF-7 cells were plated into a $35 \mathrm{~mm}$ cell culture dish. CPD4 was added to the dish to a final concentration of $4.4 \times 10^{-6} \mathrm{M}$. Fluorescence images of CPD 4 at $37^{\circ} \mathrm{C}$ were taken with LSCM under $488 \mathrm{~nm} \mathrm{Ar}^{+}$laser excitation. The 60x objective was used to get the best image quality at high magnification. The laser beam was scanned in the lateral $(x-y)$ plane generating $2 \mathrm{D}$ optically sliced images comprised of $512 \times 512$ pixels with an excellent depth resolution. The objective was moved up the $z$-axis after each lateral plane scanning to obtain a series of $2 \mathrm{D}$ images with an increment $\Delta z=0.5 \mu \mathrm{m}$ $(-10 \mu \mathrm{m} \leq z \leq 20 \mu \mathrm{m})$. LSCM images were scanned at $1.65 \mathrm{~s} /$ frame. The time interval between two adjacent images was 15 min.

\section{Results and Discussion}

3.1. Subcellular Distribution of CPD4 in MCF-7 Cells. It is well known that the photodynamic efficacy and the cell death mechanism are closely associated with the localization sites of PS in cells, while the subcellular distribution of PS is determined by its transmembrane transport mode 


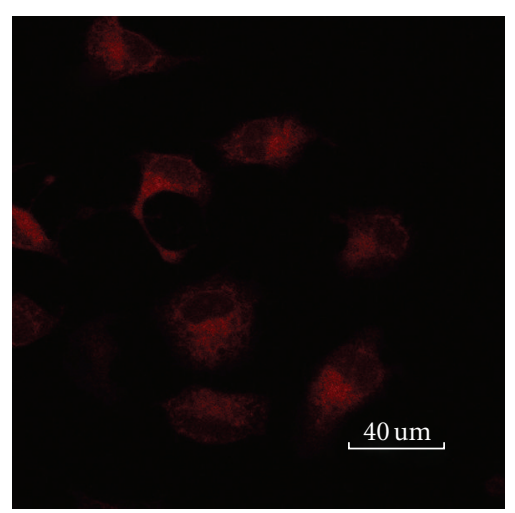

(a)

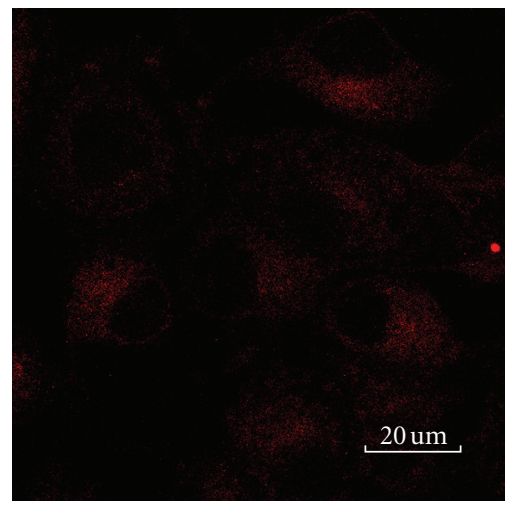

(d)

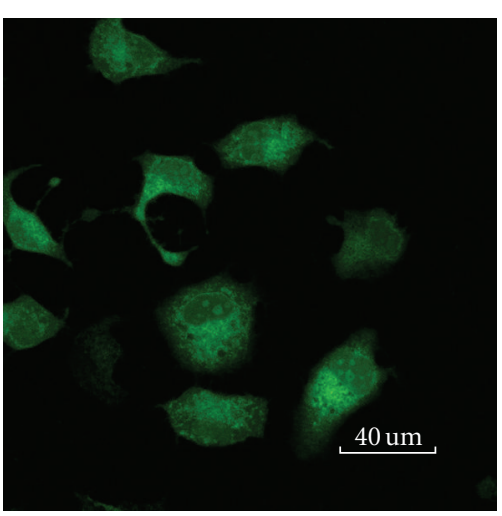

(b)

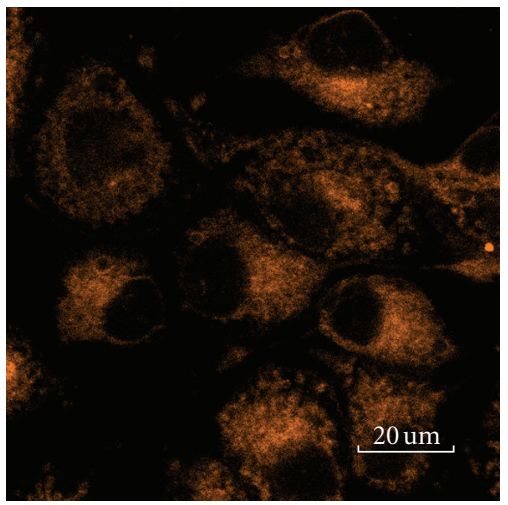

(e)

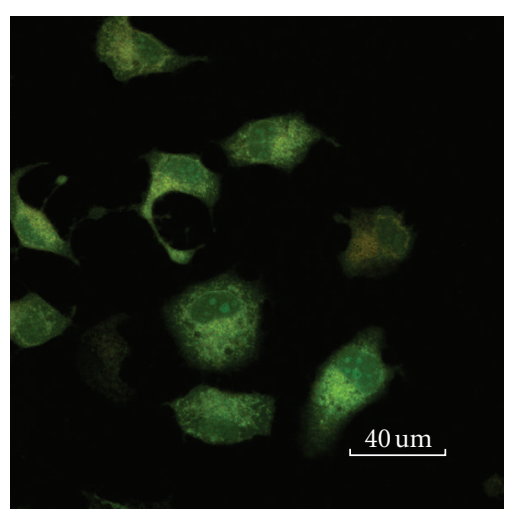

(c)

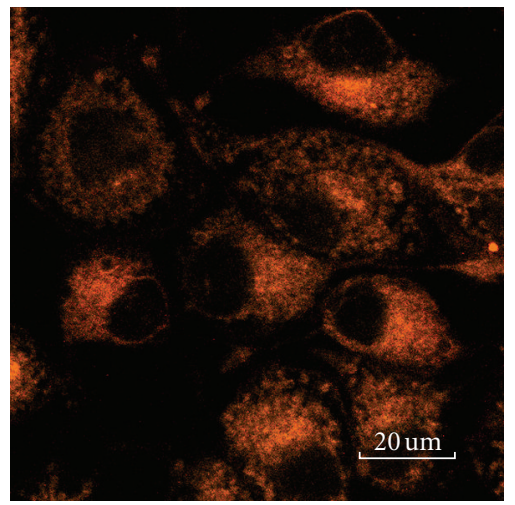

(f)

Figure 2: LSCM images of CPD4 in MCF-7 cells. (a) CPD4, (b) CPD4 and Rhodamine 123, (c) overlaid image of (a) and (b), (d) CPD4, (e) CPD4 and DiI-7, (f) overlaid image of (d) and (e).

[27]. Therefore, identifying the localization sites of CPD4 in MCF-7 cells will help us to elucidate the transport mode. Since CPD4 is fluorescent, confocal fluorescence microscopy techniques coupled with organelle-specific markers permit the determination of the subcellular distribution with high spatial resolution. In this work, the subcellular distribution of CPD4 in MCF-7 cells is studied by LSCM using two fluorescent probes-the mitochondrial probe Rhodamine 123 and the membrane probe DiI-7.

The fluorescence images of CPD4 (Figures 2(a), 2(d)), Rhodamine 123 (Figure 2(b)), and DiI-7 (Figure 2(e)) in MCF-7 cells have been assigned red, green, and orange false colors, respectively. The overlaid image of CPD4 and Rhodamine 123 shows a yellowish-green color (Figure 2(c)), while that of CPD4 and DiI-7 shows a reddish-orange color (Figure 2(f)). It can be clearly seen that there are very nice fluorescence emission overlaps between CPD4 and Rhodamine 123 and also between CPD4 and DiI-7. To further examine the degrees of these overlaps, a quantitative analysis was carried out by the calculation of correlation coefficient $r$ [39], which is a measure of the linear relationship between two variables: $r=0$ indicates no linear relationship between the two variables, while $r=1$ indicates a perfect linear relationship. The $r$ values for CPD4 with Rhodamine 123 and with DiI-7 are 0.897 and 0.627 , respectively, which support that the major localization sites of CPD4 in MCF-7 cells are on the mitochondria and membranes. It is noteworthy that similar experiments using Lyso Tracker dyes as the fluorescent probes were also done. Results confirmed that the lysosomes are not the target for CPD4 binding. The details of these observations will be reported elsewhere, together with other results. It has been pointed out that the cellular uptake of PS via diffusion transport generally leads to its accumulation on mitochondria and membranes $[25,26]$. Our present study supports the passive diffusion transport mode of CPD4. This result also implies that the binding between CPD4 and organelles helps to maintain a concentration gradient necessary for the continuous loading of PS into MCF-7 cells, which becomes a basic consideration when trying to establish a model to quantitatively describe the transmembrane transport process.

3.2. Diffusion Model of Cellular Uptake of CPD4. The above subcellular distribution study reveals that CPD4 permeates into the cells mostly by diffusion along the concentration gradient, the rates of which are determined by the diffusion coefficient. A practical way to obtain the diffusion coefficient and other parameters is to establish a diffusion model and then fit experimental data using this model. Here we propose a model based on Fick's first law of diffusion.

To facilitate understanding of the model, Figure 3 presents a cartoon illustrating the diffusion of CPD4 from 


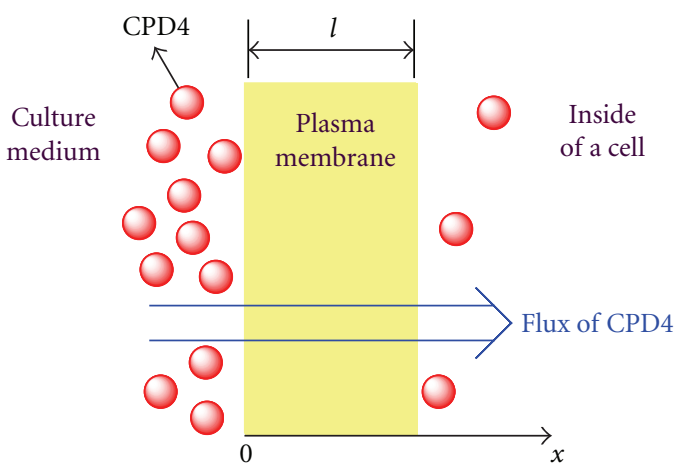

FIgUre 3: Cartoon illustrating the diffusion of CPD4 from culture medium to the inside of a cell.

culture medium to the inside of a cell. In this figure, CPD4 diffuses into the cells along the $x$-axis, where the diffusion length $l$ is equal to the thickness of the plasma membrane. Because the amount of CPD4 permeated into the cells is considerably smaller than that of CPD4 in the culture medium, we assume that the concentration of CPD4 in the culture medium, defined here as $C_{E}\left(=4.4 \times 10^{-6} \mathrm{M}\right)$, will not change during the whole diffusion process. Therefore, in our diffusion model, the total concentration of CPD4 inside a cell can be written as

$$
C_{\text {total }}(x \geq l, t)=C_{\text {free }}(t)+C_{\text {bound }}(t),
$$

where $C_{\text {free }}(t)$ and $C_{\text {bound }}(t)$ are the concentrations of intracellular free and organelle-bound CPD4, respectively. Although practically the distribution of bound CPD4 inside a cell is not even, $C_{\text {bound }}(t)$ refers to the mean concentration here for simplicity. In this equation, $x \geq l$ refers to CPD4 inside the cells as depicted in Figure 3. When CPD4 permeates into the cells from the culture medium, we suppose that it immediately binds organelles, thus becoming bound CPD4. During this process, $C_{\text {free }}(t)=0$ and $C_{\text {total }}(t)=$ $C_{\text {bound }}(t)$. As more and more CPD4 permeates into the cells, we assume, at time $t_{S}$, the binding between CPD4 and organelles reaches a saturated state. We define the CPD4 concentration at this saturated binding state as $C_{S}$, and thus we have $C_{\text {free }}\left(t \leq t_{S}\right)=0$ and $C_{\text {total }}\left(t_{S}\right)=C_{S}$.

In the case of $t \leq t_{S}$, according to Fick's first law of diffusion [40], the diffusion equation of the system can be written as

$$
J(x, t)=-D \frac{\partial C(x, t)}{\partial x}
$$

where $J$ is the flux, which represents the number of CPD4 passing through unit area of the plasma membrane within unit time, $D$ is the diffusion coefficient, and $\partial C(x, t) / \partial x$ is the concentration gradient along the $-x$ direction. At $t=0$, $C_{\text {total }}(x \geq l, t=0)=0$ and the concentration gradient of CPD4 across the membrane has a maximal value:

$$
\frac{\partial C(x, t)}{\partial x}=-\frac{\left[C_{E}-C_{\text {free }}(t)\right]}{l}=-\frac{C_{E}}{l} .
$$

Substituting (3) into (2), we get

$$
J(x=l, t)=D \frac{C_{E}}{l} .
$$

Since in our experiments the cancer cells adhere to the surface of the culture dish, we assume that, for simplicity, only the upper half of the plasma membrane can accept the permeation of CPD4, while the close contact between the lower half of the plasma membrane and the culture dish blocks CPD4 from entering into the cells. Therefore, based on (4), the total number of CPD4 permeated into a cell at the time $t$ can be written as

$$
N(t)=J(x=l, t) \frac{A}{2} t=D \frac{C_{E}}{l} \frac{A}{2} t,
$$

where $A$ is the total area of the cell membrane. Assuming $\Delta x=V / A$, where $V$ is the average volume of the cell, from the above deductions we get

$$
C_{\text {total }}(t)=C_{\text {bound }}(t)=\frac{N(t)}{V}=D \frac{C_{E}}{2 l \Delta x} t, \quad\left(t \leq t_{S}\right) .
$$

In the case of $t \geq t_{S}$, all binding sites in the organelles have been occupied by CPD4, and the concentration of free CPD4 inside the cells begins to increase from zero. Thus, we have $C_{\text {bound }}(t)=C_{S}$ and $C_{\text {free }}(t)=C_{\text {total }}(t)-C_{S}$. If the time interval $(\Delta t)$ is considerably short, we can neglect the change of the concentration gradient of CPD4 across the plasma membrane during $\Delta t$. Therefore, we have

$$
\begin{aligned}
\frac{\partial C(x, t+\Delta t)}{\partial x} \approx \frac{\partial C(x, t)}{\partial x} & =-\frac{\left[C_{E}-C_{\text {free }}(t)\right]}{l} \\
& =-\frac{\left\{C_{E}-\left[C_{\text {total }}(t)-C_{S}\right]\right\}}{l}
\end{aligned}
$$

Substituting (7) into (2), we can express $\Delta C_{\text {total }}(t, \Delta t)$, the concentration increment of intracellular CPD4 during $\Delta t$, as

$$
\begin{aligned}
\Delta C_{\text {total }}(t, \Delta t) & =\frac{\Delta N(t, \Delta t)}{V} \\
& =\frac{-D\left\{-\left(C_{E}-\left[C_{\text {total }}(t)-C_{S}\right]\right) / l\right\}(A / 2) \Delta t}{A \Delta x} \\
& =\frac{D\left\{C_{E}-\left[C_{\text {total }}(t)-C_{S}\right]\right\} \Delta t}{2 l \Delta x} .
\end{aligned}
$$

$C_{\text {total }}(t+\Delta t)$ can be calculated by using recursion as shown in the following:

$$
\begin{aligned}
C_{\text {total }}(t+\Delta t)= & C_{\text {total }}(t)+\Delta C_{\text {total }}(t, \Delta t) \\
= & \frac{D \Delta t}{2 l \Delta x}\left(C_{E}+C_{S}\right) \\
& +\left(1-\frac{D \Delta t}{2 l \Delta x}\right) C_{\text {total }}(t), \quad\left(t \geq t_{S}\right) .
\end{aligned}
$$




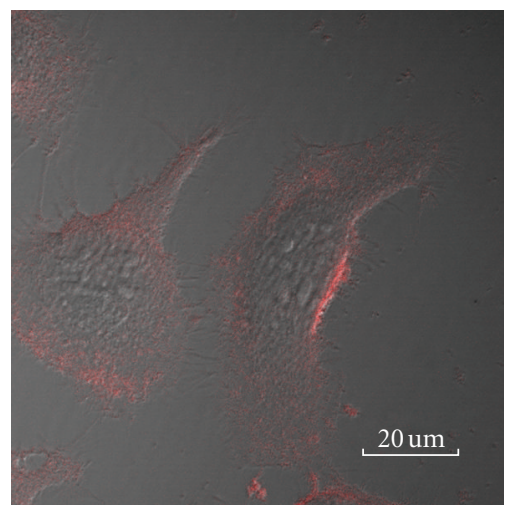

(a) $5 \mathrm{~min}$

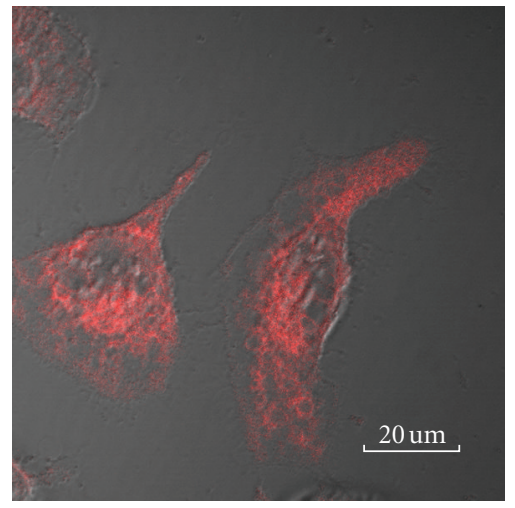

(d) $45 \mathrm{~min}$

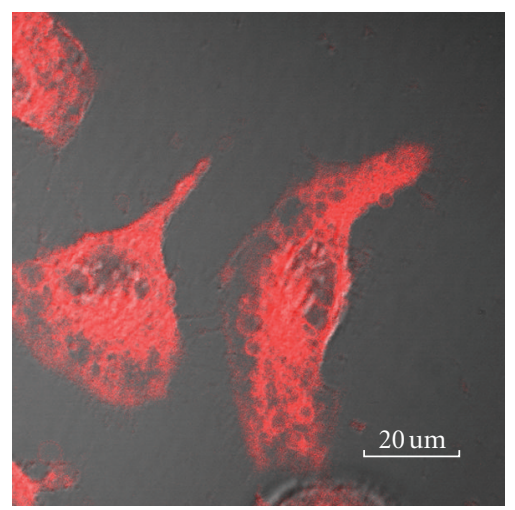

(g) $90 \mathrm{~min}$

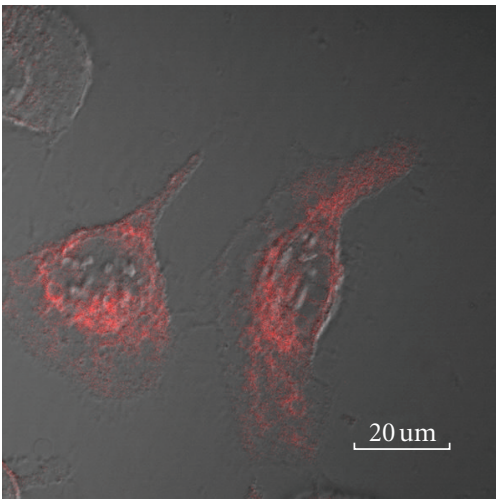

(b) $15 \mathrm{~min}$

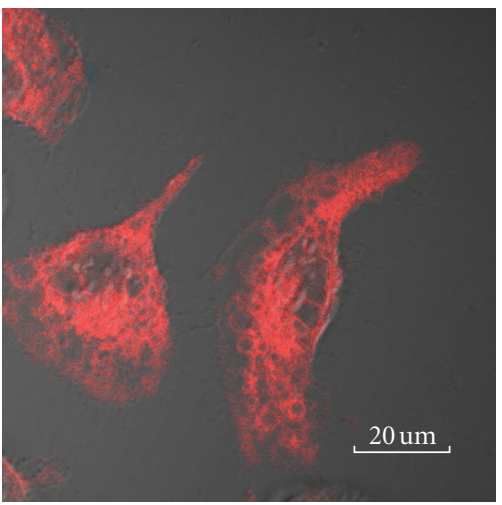

(e) $60 \mathrm{~min}$

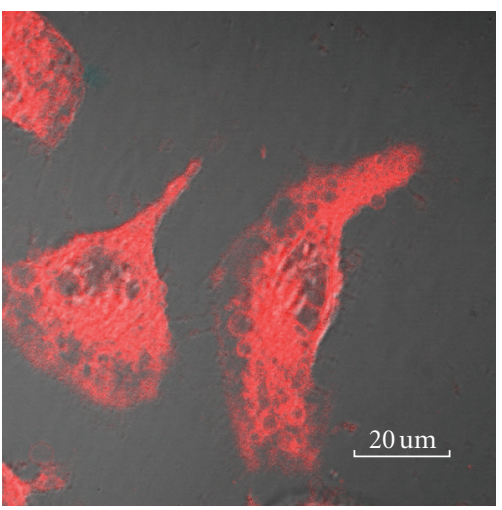

(h) $105 \mathrm{~min}$

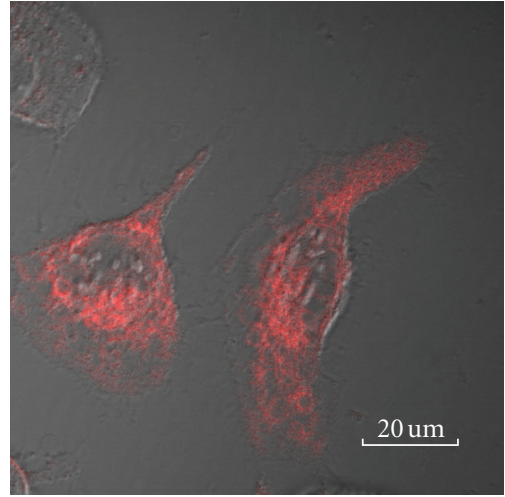

(c) $30 \mathrm{~min}$

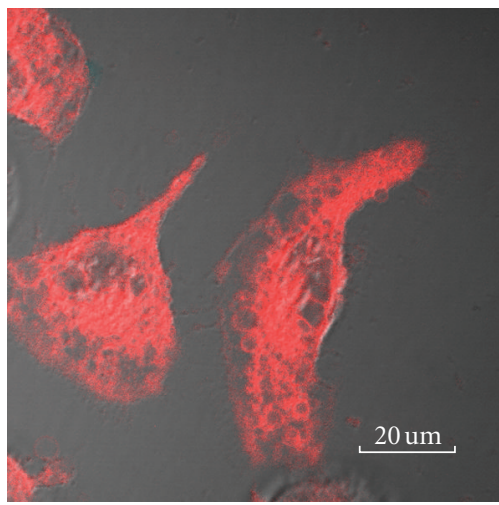

(f) $75 \mathrm{~min}$

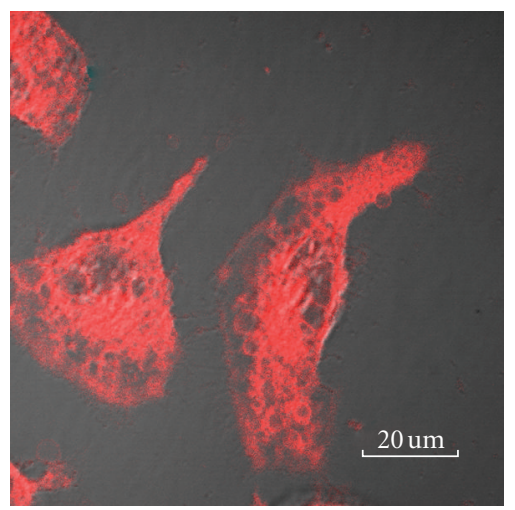

(i) $120 \mathrm{~min}$

FIgURE 4: LSCM images of CPD4 uptake in MCF-7 cells at different incubation times.

Combining (6) and (9), we now get the equations for the diffusion kinetics model, which can be used to calculate the diffusion coefficient and other parameters:

$$
C_{\text {total }}(t)=\left\{\begin{array}{l}
\frac{C_{E}}{2 l \Delta x} t, \quad\left(t \leq t_{S}\right) \\
\frac{D \Delta t}{2 l \Delta x}\left(C_{E}+C_{S}\right) \\
\quad+\left(1-\frac{D \Delta t}{2 l \Delta x}\right) C_{\text {total }}(t-\Delta t), \quad\left(t-\Delta t \geq t_{S}\right) .
\end{array}\right.
$$

In the next section, we will carry out the experimental study of the cellular uptake kinetics of CPD4 and fit the data using (10).

3.3. Kinetics of CPD4 Uptake in MCF-7 Cells. Imaging the distributions of CPD4 in MCF-7 cells at different incubation times is done using three-dimensional LSCM. Figure 4 shows the observed images, which are recorded on the same focal plane of $z=0$. The variation of the amount of CPD4 is proportional to the change of its relative fluorescence intensities [41]. Figure 4(a) clearly shows that CPD4 accumulates on the plasma membrane of MCF-7 cells 5 min after adding 
CPD4. As the incubation time increases from 15 to $45 \mathrm{~min}$, CPD4 keeps on permeating into the cells and migrates from the plasma membrane to various sensitive stores within the cells such as the mitochondria and the nucleus membranes (Figures 4(b)-4(d)). From 60 to $90 \mathrm{~min}$, the amount of CPD4 localized on these cell organelles increases obviously (Figures 4(e)-4(g)). After 90 minutes, the fluorescence intensities of CPD4 in these images remain unchanged indicating that a saturated binding state has been reached (Figures 4(h)-4(i)).

The total concentration of CPD4 at time $t$ can be determined by fluorescence measurement:

$$
C_{\text {total }}(t)=\frac{I_{\text {total }}(t)}{I_{E}} C_{E},
$$

where $I_{\text {total }}(t)$ and $I_{E}$ represent the average of relative fluorescence intensities of CPD4 inside the cells and in the culture medium, respectively, and can be obtained by summing the fluorescence intensities of each corresponding pixel in all scanned images. $C_{E}\left(=4.4 \times 10^{-6} \mathrm{M}\right)$ is known; thus $C_{\text {total }}(t)$ at different incubation time $t$ can be calculated using (11) and the results are shown in Figure 5.

Using (10), cellular uptake and distribution parameters of CPD 4 can be acquired by fitting the data given in Figure 5. In the fitting, $l=7.5 \mathrm{~nm}[42], \Delta x=3.33 \mu \mathrm{m}$, and $\Delta t=1 \mathrm{~s}$ are used. Fitting procedures are repeated iteratively until the degree of fitting exceeds 0.99 . The fitting curve is also shown in Figure 5. From the fitting, we obtain that $D$ is $8.11 \times 10^{-16} \mathrm{~m}^{2} / \mathrm{s}, C_{S}$ is $4.018 \times 10^{-4} \mathrm{M}$, and the CPD4 concentration at the diffusion equilibrium state inside the cells is $4.062 \times 10^{-4} \mathrm{M}$. These results indicate that the concentration of intracellular CPD4 is much higher than that of CPD4 in the culture medium $\left(4.4 \times 10^{-6} \mathrm{M}\right)$. The number of binding sites in an MCF-7 cell can be calculated: $N=C_{S} V=1.01 \times 10^{9}$. It is obvious that the rates of cellular uptake of CPD4 before and after reaching the saturated binding state are quite different: the former is equal to the slope of the fitting curve, $4.3 \times 10^{-6} \mathrm{M} / \mathrm{min}\left(=C_{S} / t_{S}\right.$, from which we can also calculate the time of reaching the saturated binding state: $t_{S}=93.7 \mathrm{~min}$ ); the latter is $5.5 \times 10^{-7} \mathrm{M} / \mathrm{min}$, which is attributed to the concentration difference of free CPD4 on both sides of the plasma membrane. It is noteworthy that the fitting curve shows a smooth curvature (the inset of Figure 5), which indicates the choice of $\Delta t$ is appropriate. Taken together, these results suggest that (1) the diffusion kinetics model established here can be used to quantitatively describe the transport process of CPD4 through the plasma membrane of MCF-7 cells and (2) a large number of CPD4 can bind inside MCF-7 cells, which is an important factor in achieving optimal effect of PDT.

\section{Conclusions}

In summary, the kinetics of photosensitizer CPD4 uptake in MCF-7 human breast cancer cells is described by a diffusion kinetics model and experimentally investigated using LSCM. CPD4 permeated into MCF-7 cells with increasing incubation time, which was followed by the binding between CPD4 and organelles. Subcellular distribution study revealed that

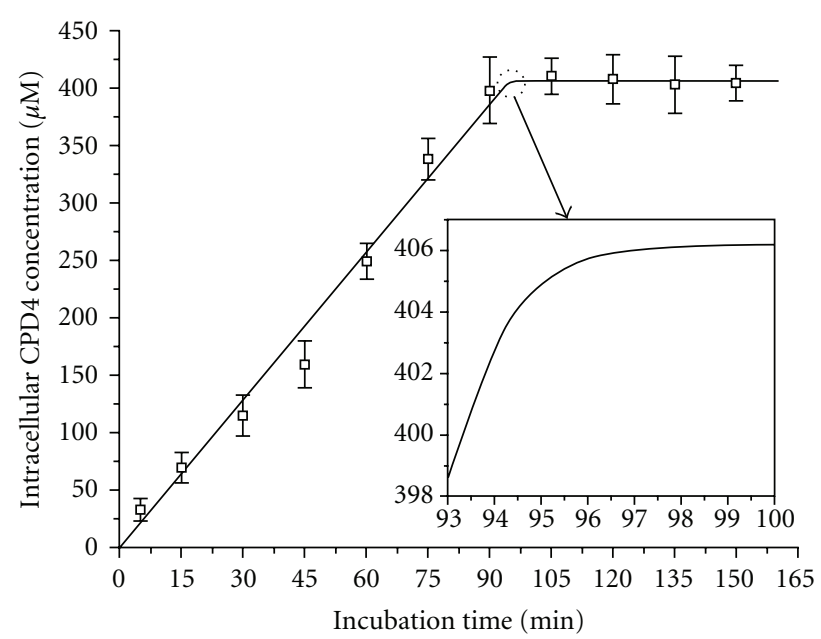

FIGURE 5: Total concentration of CPD4 in MCF-7 cells as a function of the incubation time. $\square$ : total concentration of CPD4 calculated from the experimental data; - : the fitting curve.

CPD4 was primarily localized on the mitochondria and cell membranes, supporting that the mode of transmembrane transport was diffusion. A kinetics model describing CPD4 passing through the plasma membrane of MCF-7 cells was proposed based on Fick's first law of diffusion. The kinetics of cellular uptake of CPD4 was studied by three-dimensional LSCM. By fitting fluorescence intensity data using the above model, important cellular uptake and distribution parameters were obtained: the diffusion coefficient of CPD4 across the plasma membrane was $8.11 \times 10^{-16} \mathrm{~m}^{2} / \mathrm{s}$; the concentration of organelle-bound CPD4 at the saturated binding state was $4.018 \times 10^{-4} \mathrm{M}$; the concentration of CPD4 at the diffusion equilibrium state inside the cells was $4.062 \times$ $10^{-4} \mathrm{M}$; the average number of CPD4 binding sites in an MCF-7 cell is $1.01 \times 10^{9}$; the rates of cellular uptake of CPD4 before and after reaching the saturated binding state were $4.3 \times 10^{-6}$ and $5.5 \times 10^{-7} \mathrm{M} / \mathrm{min}$, respectively. These results indicate that the diffusion kinetics model established in this work can be used to obtain quantitative information about the cellular transport process of PS, which will contribute to the better understanding of PDT mechanisms and the development of new PS with optimized interactions with target cells and tissues. Future works that are being planned include investigating the physiochemical properties of CPD4 and performing concentration-dependent uptake experiments using CPD4 as well as other compounds with specific known properties.

\section{Authors' Contribution}

P. Chen and J.-Z. Yao contributed equally to this work.

\section{Acknowledgments}

The authors gratefully acknowledge Professor Tong and Mr. Qiao (Cancer Hospital of Tianjin Medical University, Tianjin, China) for offering MCF-7 human breast cancer 
cells. This research was supported by National Natural Science Foundation of China (nos. 60508004, 60778043, 30371737, and 81172950), the National High Technology Research and Development Program ("863" Program) of China (no. 2011AA030205), and Tianjin Municipal Science and Technology Commission (no. 08ZCDFGX09400).

\section{References}

[1] R. M. El-Shishtawy, "Functional dyes, and some hi-tech applications," International Journal of Photoenergy, vol. 2009, Article ID 434897, 21 pages, 2009.

[2] D. Frackowiak, K. Wiktorowicz, A. Planner, A. Waszkowiak, and R. M. Ion, "The phthalocyanines applications in photodynamic therapy investigated by time-resolved and steady-state photothermal methods," International Journal of Photoenergy, vol. 4, no. 2, pp. 51-56, 2002.

[3] A. E. O'Connor, W. M. Gallagher, and A. T. Byrne, "Porphyrin and nonporphyrin photosensitizers in oncology: preclinical and clinical advances in photodynamic therapy," Photochemistry and Photobiology, vol. 85, no. 5, pp. 1053-1074, 2009.

[4] S. Yano, S. Hirohara, M. Obata et al., "Current states and future views in photodynamic therapy," Journal of Photochemistry and Photobiology C, vol. 12, no. 1, pp. 46-67, 2011.

[5] E. Paszko, C. Ehrhardt, M. O. Senge, D. P. Kelleher, and J. V. Reynolds, "Nanodrug applications in photodynamic therapy," Photodiagnosis and Photodynamic Therapy, vol. 8, no. 1, pp. 14-29, 2011.

[6] J. P. Celli, B. Q. Spring, I. Rizvi et al., "Imaging and photodynamic therapy: mechanisms, monitoring, and optimization," Chemical Reviews, vol. 110, no. 5, pp. 2795-2838, 2010.

[7] R. R. Allison and C. H. Sibata, "Oncologic photodynamic therapy photosensitizers: a clinical review," Photodiagnosis and Photodynamic Therapy, vol. 7, no. 2, pp. 61-75, 2010.

[8] D. E. J. G. J. Dolmans, D. Fukumura, and R. K. Jain, "Photodynamic therapy for cancer," Nature Reviews Cancer, vol. 3, no. 5, pp. 380-387, 2003.

[9] T. J. Dougherty, C. J. Gomer, B. W. Henderson et al., "Photodynamic therapy," Journal of the National Cancer Institute, vol. 90, no. 12, pp. 889-905, 1998.

[10] B. Hu, Y. He, and Z. Liu, "NIR area array CCD-based singlet oxygen luminescence imaging for photodynamic therapy," Journal of Physics: Conference Series, vol. 277, no. 1, Article ID 012011, 2011.

[11] X. Ragàs, M. Agut, and S. Nonell, "Singlet oxygen in Escherichia coli: new insights for antimicrobial photodynamic therapy," Free Radical Biology and Medicine, vol. 49, no. 5, pp. 770-776, 2010.

[12] J. Zhang, K.-L. Wong, W.-K. Wong, N.-K. Mak, D. W. J. Kwong, and H.-L. Tam, "Two-photon induced luminescence, singlet oxygen generation, cellular uptake and photocytotoxic properties of amphiphilic $\mathrm{Ru}(\mathrm{II})$ polypyridyl-porphyrin conjugates as potential bifunctional photodynamic therapeutic agents," Organic \& Biomolecular Chemistry, vol. 9, no. 17, pp. 6004-6010, 2011.

[13] S. Hackbarth, J. Schlothauer, A. Preuß, and B. Röder, "New insights to primary photodynamic effects-singlet oxygen kinetics in living cells," Journal of Photochemistry and Photobiology B, vol. 98, no. 3, pp. 173-179, 2010.

[14] K. K. H. Wang, J. C. Finlay, T. M. Busch, S. M. Hahn, and T. C. Zhu, "Explicit dosimetry for photodynamic therapy: macroscopic singlet oxygen modeling," Journal of Biophotonics, vol. 3, no. 5-6, pp. 304-318, 2010.
[15] J. Fuchs and J. Thiele, "The role of oxygen in cutaneous photodynamic therapy," Free Radical Biology and Medicine, vol. 24, no. 5, pp. 835-847, 1998.

[16] J. M. Fernandez, M. D. Bilgin, and L. I. Grossweiner, "Singlet oxygen generation by photodynamic agents," Journal of Photochemistry and Photobiology B, vol. 37, no. 1-2, pp. 131140, 1997.

[17] Y. Cheng, A. C. Samia, J. D. Meyers, I. Panagopoulos, B. Fei, and C. Burda, "Highly efficient drug delivery with gold nanoparticle vectors for in vivo photodynamic therapy of cancer," Journal of the American Chemical Society, vol. 130, no. 32, pp. 10643-10647, 2008.

[18] P. Zhang, W. Steelant, M. Kumar, and M. Scholfield, "Versatile photosensitizers for photodynamic therapy at infrared excitation," Journal of the American Chemical Society, vol. 129, no. 15, pp. 4526-4527, 2007.

[19] M. B. Vrouenraets, G. W. M. Visser, G. B. Snow, and G. A. M. S. Van Dongen, "Basic principles, applications in oncology and improved selectivity of photodynamic therapy," Anticancer Research, vol. 23, no. 1 B, pp. 505-522, 2003.

[20] Y. N. Konan, R. Gurny, and E. Allémann, "State of the art in the delivery of photosensitizers for photodynamic therapy," Journal of Photochemistry and Photobiology B, vol. 66, no. 2, pp. 89-106, 2002.

[21] Y. G. Qiang, C. M. N. Yow, and Z. Huang, "Combination of photodynamic therapy and immunomodulation: current status and future trends," Medicinal Research Reviews, vol. 28, no. 4, pp. 632-644, 2008.

[22] J. Moan and K. Berg, "The photodegradation of porphyrins in cells can be used to estimate the lifetime of singlet oxygen," Photochemistry and Photobiology, vol. 53, no. 4, pp. 549-553, 1991.

[23] H. Mojzisova, S. Bonneau, and D. Brault, "Structural and physico-chemical determinants of the interactions of macrocyclic photosensitizers with cells," European Biophysics Journal, vol. 36, no. 8, pp. 943-953, 2007.

[24] Z. Xiao, C. B. Hansen, T. M. Allen, G. G. Miller, and R. B. Moore, "Distribution of photosensitizers in bladder cancer spheroids: implications for intravesical instillation of photosensitizers for photodynamic therapy of bladder cancer," Journal of Pharmacy and Pharmaceutical Sciences, vol. 8, no. 3, pp. 536-543, 2005.

[25] W. G. Roberts and M. W. Berns, "In vitro photosensitization. I. Cellular uptake and subcellular localization of mono-Laspartyl chlorin $\mathrm{e}_{6}$, chloro-aluminum sulfonated phthalocyanine, and photofrin II," Lasers in Surgery \& Medicine, vol. 9, no. 2, pp. 90-101, 1989.

[26] W. D. Dai, X. S. Li, J. Zeng, F. G. Liu, and Y. Gu, "Influence of combination form of hematoporphyrin monomethyl ether with different kinds of serum protein on its intracellular distribution," Chinese Journal of Laser Medicine \& Surgery, vol. 15, no. 3, pp. 137-140, 2006.

[27] T. Osaki, S. Takagi, Y. Hoshino, M. Okumura, and T. Fujinaga, "Intracellular localization and concentration as well as photodynamic effects of benzoporphyrin derivative monoacid ring A in four types of rodent tumor cells," Cancer Letters, vol. 243, no. 2, pp. 281-292, 2006.

[28] C. A. Robertson, D. H. Evans, and H. Abrahamse, "Photodynamic therapy (PDT): a short review on cellular mechanisms and cancer research applications for PDT," Journal of Photochemistry and Photobiology B, vol. 96, no. 1, pp. 1-8, 2009.

[29] A. A. Stratonnikov, A. Y. Douplik, and V. B. Loschenov, "Oxygen consumption and photobleaching in whole blood 
incubated with photosensitizer induced by laser irradiation,” Laser Physics, vol. 13, no. 1, pp. 1-21, 2003.

[30] J. Braga, J. G. McNally, and M. Carmo-Fonseca, "A reactiondiffusion model to study RNA motion by quantitative fluorescence recovery after photobleaching," Biophysical Journal, vol. 92, no. 8, pp. 2694-2703, 2007.

[31] L. Wawrezinieck, P. F. Lenne, D. Marguet, and H. Rigneault, "Fluorescence correlation spectroscopy to determine diffusion laws: application to live cell membranes," in Biophotonics Micro- and Nano-Imaging, vol. 5462 of Proceedings of SPIE, pp. 92-102, Strasbourg, France.

[32] E. D. Sternberg, D. Dolphin, and C. Brückner, "Porphyrinbased photosensitizers for use in photodynamic therapy," Tetrahedron, vol. 54, no. 17, pp. 4151-4202, 1998.

[33] H. Ali and J. E. Van Lier, "Metal complexes as photo- and radiosensitizers," Chemical Reviews, vol. 99, no. 9, pp. 23792450, 1999.

[34] R. Dai, R. Shoemaker, D. Farrens, M. J. Han, C. S. Kim, and P. S. Song, "Characterization of silkworm chlorophyll metabolites as an active photosensitizer for photodynamic therapy," Journal of Natural Products, vol. 55, no. 9, pp. 1241-1251, 1992.

[35] J. Yao, W. Chen, X. He et al., "Synthesis and photosensitizing abilities as well as tumor-photobiological activities of chlorin F methyl ether," Acta Pharmaceutica Sinica, vol. 35, no. 1, pp. 63-66, 2000.

[36] P. Chen, J. Z. Yao, G. Q. Tang et al., "Studies on photodynamic effects of $\mathrm{CPD}_{1}$ on HL-60 cells in vitro," Journal of Optoelectronics.Laser, vol. 13, no. 9, pp. 972-975, 2002.

[37] P. Chen and J. Z. Yao, "Studies on fluorescence emission properties and quantum yield of singlet oxygen photosensitized by chlorin photosensitizers," Journal of Optoelectronics - Laser, vol. 13, no. 10, pp. 1081-1086, 2002.

[38] P. D. Zhao, P. Chen, G. Q. Tang et al., "Photophysical properties of the chlorophyll derivative under two-photon excitation," Journal of Optoelectronics - Laser, vol. 16, no. 7, pp. 881886, 2005.

[39] N. S. Trivedi, H. W. Wang, A. L. Nieminen, N. L. Oleinick, and J. A. Izatt, "Quantitative analysis of Pc 4 localization in mouse lymphoma (LY-R) cells via double-label confocal fluorescence microscopy," Photochemistry and Photobiology, vol. 71, no. 5, pp. 634-639, 2000.

[40] P. W. Atkins, Physical Chemistry, Oxford University Press, Oxford, UK, 2nd edition, 1982.

[41] B. M. Aveline, T. Hasan, and R. W. Redmond, "The effects of aggregation, protein binding and cellular incorporation on the photophysical properties of benzoporphyrin derivative monoacid ring A (BPDMA)," Journal of Photochemistry and Photobiology B, vol. 30, no. 2-3, pp. 161-169, 1995.

[42] Z. G. Shen and D. C. Cui, "Plasma membrane and cell surface," in Cell Biology, p. 58, China Agriculture Press, Beijing, China, 2003. 


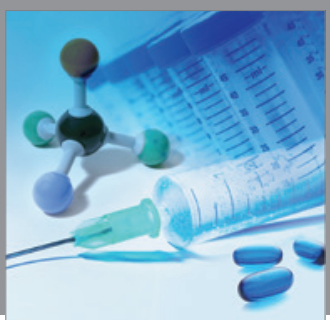

International Journal of

Medicinal Chemistry

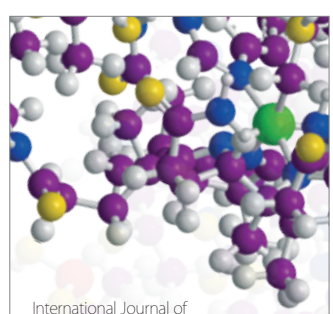

Carbohydrate Chemistry

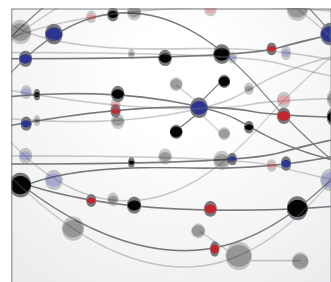

The Scientific World Journal
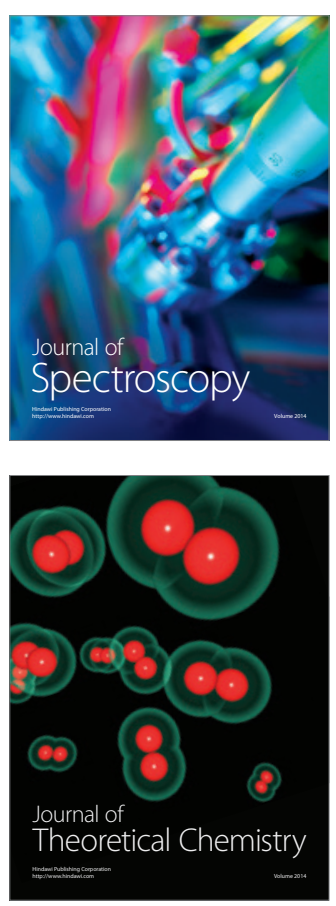
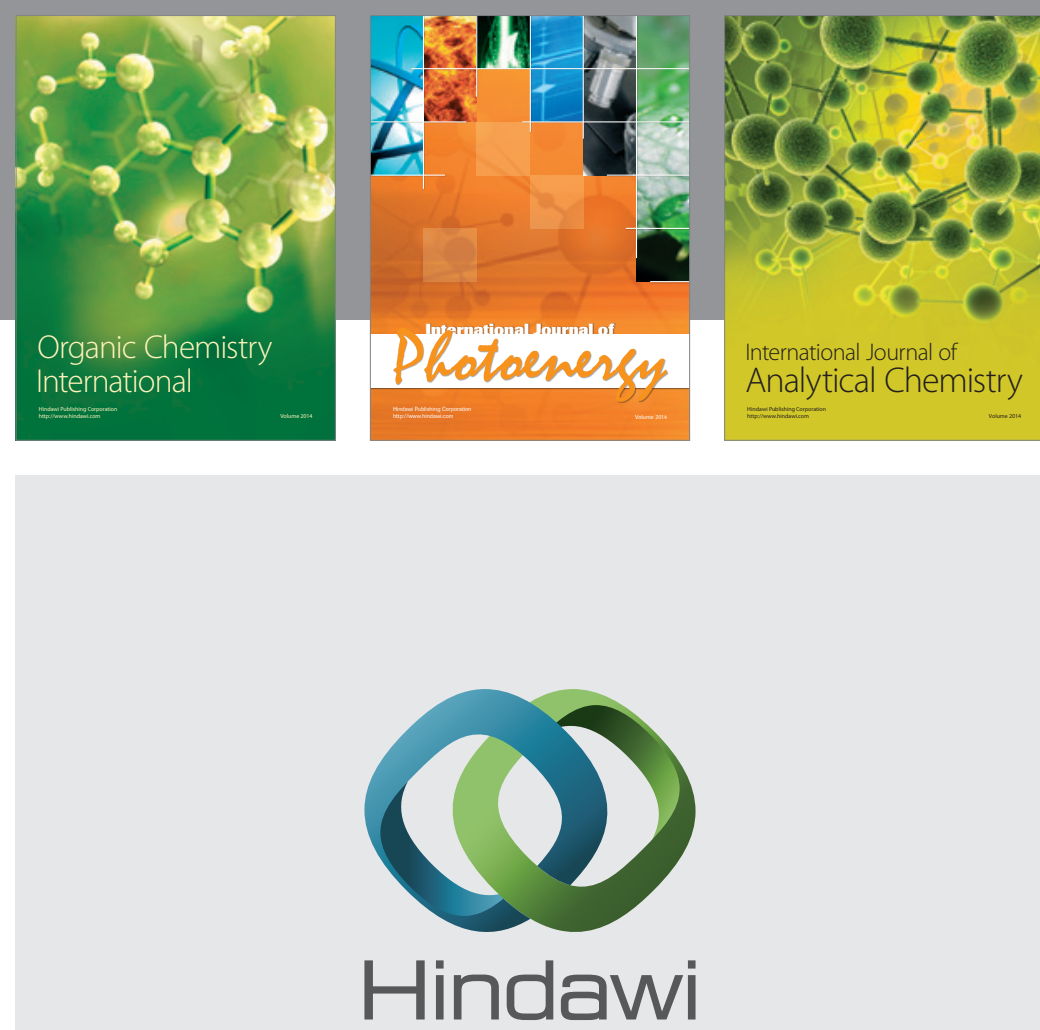

Submit your manuscripts at

http://www.hindawi.com
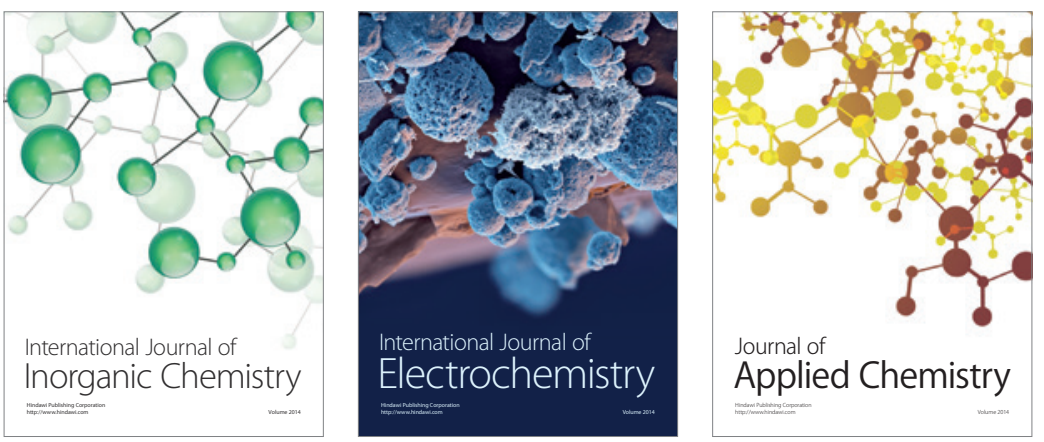

Journal of

Applied Chemistry
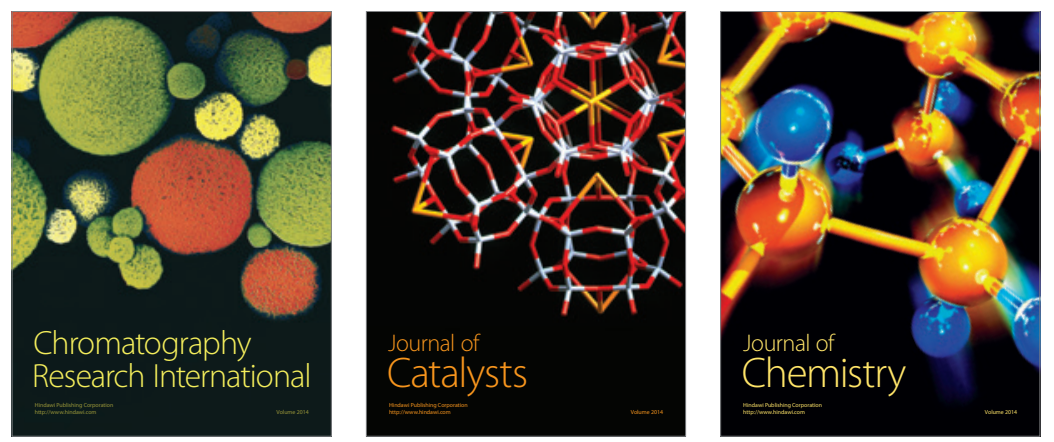
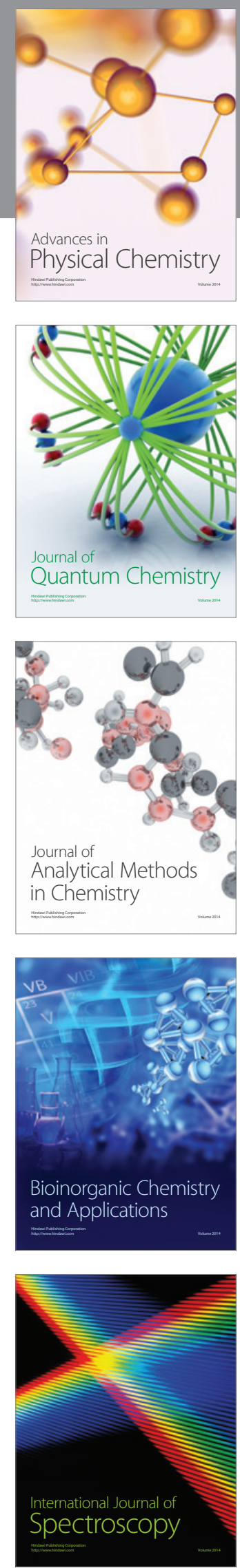